\title{
Observations géomorphologiques sur les méga-blocs du secteur sud-est de la batture argileuse de la baie à l'Orignal, au parc du Bic, dans le Bas-Saint-Laurent (Québec) Geomorphologic Observations on Mega-Boulders in the Southern Area of the Clayey Tidal-Flat of the Baie à L'Orignal, Parc du Bic, Bas-Saint-Laurent (Québec)
}

\section{Jean-Claude Dionne}

\section{Volume 57, numéro 1, 2003}

URI : https://id.erudit.org/iderudit/010333ar

DOI : https://doi.org/10.7202/010333ar

\section{Aller au sommaire du numéro}

Éditeur(s)

Les Presses de l'Université de Montréal

ISSN

0705-7199 (imprimé)

1492-143X (numérique)

Découvrir la revue

Citer cette note

Dionne, J.-C. (2003). Observations géomorphologiques sur les méga-blocs du secteur sud-est de la batture argileuse de la baie à l'Orignal, au parc du Bic, dans le Bas-Saint-Laurent (Québec). Géographie physique et Quaternaire, 57(1), 95-101. https://doi.org/10.7202/010333ar

\section{Résumé de l'article}

De nombreux méga-blocs épars caractérisent la batture argileuse de la baie à l'Orignal, dans le parc du Bic, sur la rive sud de l'estuaire maritime du Saint-Laurent. Des 160 blocs mesurés, 46,3\% sont des erratiques provenant du Bouclier laurentidien sur la côte nord et 53,7 \% des éléments appalachiens provenant, pour la majorité, des crêtes rocheuses en bordure de la côte sud. Sur tous les blocs mesurés, la longueur (axe $a$ ) excédait $100 \mathrm{~cm}$ et le poids du plus petit (un précambrien), une tonne, alors que le plus gros (un appalachien) pesait 76 tonnes et mesurait 565 × 350 × $205 \mathrm{~cm}$. Situés en surface, la majorité des méga-blocs sont susceptibles d'être déplacés par les radeaux de glace. Les blocs le plus souvent déplacés sont ceux du marais à Spartine alterniflore (schorre inférieur). Des indices de déplacement ont en effet été observés sur près de $32 \%$ des blocs ; le plus grand déplacement, observé sur un gneiss d'environ 6 tonnes, atteint $6 \mathrm{~m}$.
Tous droits réservés @ Les Presses de l'Université de Montréal, 2004
Ce document est protégé par la loi sur le droit d'auteur. L’utilisation des services d’Érudit (y compris la reproduction) est assujettie à sa politique d'utilisation que vous pouvez consulter en ligne.

https://apropos.erudit.org/fr/usagers/politique-dutilisation/ 


\title{
OBSERVATIONS GÉOMORPHOLOGIQUES SUR LES MÉGA-BLOCS DU SECTEUR SUD-EST DE LA BATTURE ARGILEUSE DE LA BAIE À L'ORIGNAL, AU PARC DU BIC, DANS LE BAS-SAINT-LAURENT (QUÉBEC)
}

\author{
Jean-Claude DIONNE, Département de géographie et Centre d'études nordiques, Université Laval, Québec G1K 7P4.
}

RÉSUMÉ De nombreux méga-blocs épars caractérisent la batture argileuse de la baie à l'Orignal, dans le parc du Bic, sur la rive sud de l'estuaire maritime du Saint-Laurent. Des 160 blocs mesurés, $46,3 \%$ sont des erratiques provenant du Bouclier laurentidien sur la côte nord et $53,7 \%$ des éléments appalachiens provenant, pour la majorité, des crêtes rocheuses en bordure de la côte sud. Sur tous les blocs mesurés, la longueur (axe a) excédait $100 \mathrm{~cm}$ et le poids du plus petit (un précambrien), une tonne, alors que le plus gros (un appalachien) pesait 76 tonnes et mesurait $565 \times 350 \times 205 \mathrm{~cm}$. Situés en surface, la majorité des méga-blocs sont susceptibles d'être déplacés par les radeaux de glace. Les blocs le plus souvent déplacés sont ceux du marais à Spartine alterniflore (schorre inférieur). Des indices de déplacement ont en effet été observés sur près de $32 \%$ des blocs ; le plus grand déplacement, observé sur un gneiss d'environ 6 tonnes, atteint $6 \mathrm{~m}$.

\section{INTRODUCTION}

Les régions du Saint-Laurent, notamment les grandes battures argileuses de la rive sud, sont généralement caractérisées par la présence de milliers de cailloux de taille et de nature variées comprenant, en général, environ $50 \%$ d'éléments laurentidiens (Dionne, 1972). Certains (Fortin et Belzile, 1996, par exemple) attribuent encore la présence de ces erratiques aux glaciers venus du nord au cours de la dernière période glaciaire, dite du Wisconsinien. Or, l'absence généralisée de till ou de dépôts glaciaires en bordure du littoral actuel, le faible pourcentage d'éléments laurentidiens à l'intérieur des terres en dehors de la zone couverte par la Mer de Goldthwait (Rappol, 1993) et la présence de dépôts marins argileux à l'emplacement des battures capitonnées de blocs (Dionne, 1966) ont permis d'établir que les erratiques laurentidiens avaient plutôt été transportés et délestés par les glaces flottantes annuelles et les icebergs (Dionne, 1972, 1979, 2001a, 2002a, b; Dionne et Poitras, 1998a, b).

La présente contribution concerne les méga-blocs du secteur sud-est de la baie à l'Orignal, dans le parc du Bic. L'étude examine la nature, la taille et la mobilité des méga-blocs de la partie supérieure de la batture.

Manuscrit reçu le 23 avril 2003 ; manuscrit révisé accepté le 30 mai 2003 (publié en octobre 2004)

Adresse électronique : Dionne.Morissette@globetrotter.net
ABSTRACT Geomorphologic observations on mega-boulders in the southern area of the clayey tidal-flat of the Baie à l'Orignal, Parc du Bic, Bas-Saint-Laurent (Québec). Many mega-boulders are scattered on the clayey tidal flat in the Parc du Bic, on the south shore of the lower St. Lawrence estuary. Of the 160 boulders measured, $46.3 \%$ are erratics from the Laurentidian Shield on the north shore, and $53.7 \%$ are Appalachian clasts mainly from the coastal rock ridges on the south shore. All boulders measured exceeded $100 \mathrm{~cm}$ and the weight of the smallest (a Precambrian) was one metric ton whereas the largest (an Appalachian) weighed 76 tons and measured $565 \times 350 \times 205 \mathrm{~cm}$. Most mega-boulders resting on the surface of the tidal flat are likely to be moved freely by ice blocks. Those in the low marsh are more exposed and move more frequently. Evidence of displacement has been observed for $32 \%$ of the mega-boulders in the low marsh; the longest displacement of a gneiss boulder weighing 6 tons was $6 \mathrm{~m}$.

\section{SITUATION GÉOGRAPHIQUE ET CARACTÉRISTIQUES DU MILIEU}

La baie à l'Orignal se trouve dans la partie centrale du parc du Bic, sur la rive sud du Saint-Laurent estuarien $\left(68^{\circ} 47^{\prime} \mathrm{O}\right.$, $48^{\circ} 30^{\prime} \mathrm{N}$ ), à environ $25 \mathrm{~km}$ au SO de Rimouski et $280 \mathrm{~km}$ au NE de Québec (fig. 1). La baie à l'Orignal forme un vaste rentrant entouré de plusieurs crêtes rocheuses appalachiennes dont la plupart excèdent $100 \mathrm{~m}$ d'altitude, le point le plus élevé étant le pic Champlain (346 m), à Saint-Fabien.

Allongées et orientées SO-NE, les crêtes rocheuses sont des fragments résiduels de plis déversés vers le NO et constitués de roches sédimentaires d'âge cambro-ordovicien comprenant des conglomérats calcaires, des grès quartzitiques, calcaires ou de type grauwacke, ainsi que des schistes ${ }^{1}$ gris, rouges et verts appelés maintenant mudstone, claystone et siltstone (Lajoie, 1971; Tremblay, 1986; Favereau, 1988). Des dépressions de tailles variées séparent les crêtes rocheuses qui forment l'ossature du relief côtier. Partiellement comblées par les dépôts fins (argile et limon) et grossiers (sable et gravier) de la Mer de Goldthwait, qui a submergé les lieux entre 12,5 et $9 \mathrm{ka}$, ces dépressions correspondent à des rentrants (anses et baies).

1. Le vocable «pélite», parfois utilisé, caractérise la granulométrie; ce n'est pas le nom d'une roche. Selon cette classification basée sur la texture, les grès sont des «arénites " et les conglomérats, des «rudites». 


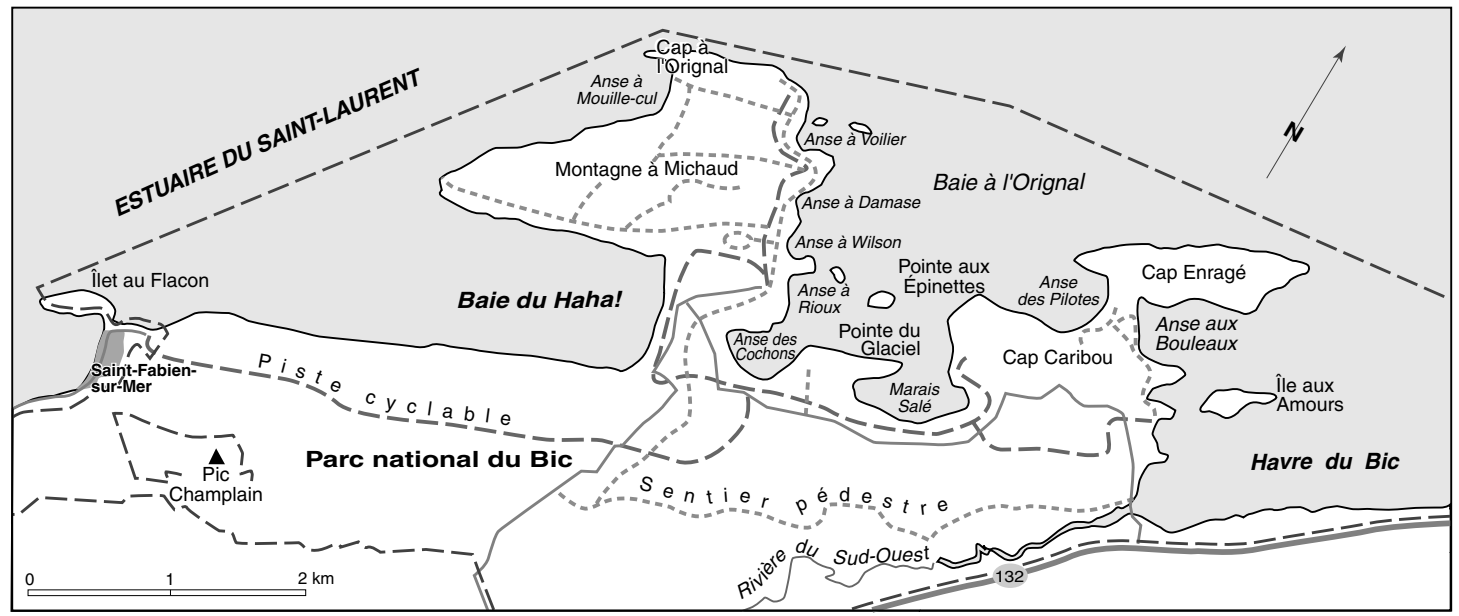

FIGURE 1. Le parc du Bic : localisation et toponymie.

La baie à l'Orignal, le plus vaste rentrant du complexe avec le havre du Bic et la baie du Haha (voir l'Appendice), est essentiellement argileuse et couverte, ici et là, de minces placages sableux et caillouteux. Dans le secteur SE, du côté NE du mont Chocolat, la petite anse abritée, appelée «Marais Salé ", a elle aussi un substrat argileux. Dans le secteur externe, en dessous du niveau moyen de la mer (zéro géodésique), la batture argileuse est dénudée et recouverte d'un mince dépôt sableux, alors que, dans le secteur au-dessus du niveau moyen de la mer, s'étend un vaste marais (schorre) à Spartine alterniflore (Spartina alterniflora) développé directement sur la surface argileuse et voilé d'une mince $( \pm 5 \mathrm{~cm})$ couche vaseuse et caillouteuse (fig. 2). À la partie supérieure, le marais à marelles, inondé seulement lors des grandes marées, est formé d'un dépôt intertidal vaseux de 50 à $80 \mathrm{~cm}$ d'épaisseur qui repose sur la surface argileuse. Les nombreux méga-blocs épars qui caractérisent ce secteur de la baie à l'Orignal ont fait l'objet d'un relevé à l'été 2002. Ont été mesurés seulement les blocs dont la taille (axe a) excédait $100 \mathrm{~cm}$. En conséquence, le nombre de blocs retenus représente un faible pourcentage des cailloux concentrés dans ce secteur, pour la plupart petits et formant des dallages.

\section{NATURE LITHOLOGIQUE DES MÉGA-BLOCS}

Les méga-blocs de la batture argileuse du secteur sud-est de la baie à l'Orignal, en particulier du secteur du marais à Spartine alterniflore (fig. 3) et de l'estran dénudé adjacent, appartiennent à deux grandes familles. II y a des éléments précambriens, ignés et métamorphiques, provenant du Bouclier laurentidien sur la côte nord du Saint-Laurent, et des éléments sédimentaires provenant principalement des formations appalachiennes d'âge cambro-ordovicien en bordure de la rive sud, notamment des crêtes rocheuses locales (fig. 4-6).

Sur les 160 méga-blocs mesurés et identifiés à la surface de la batture, $46,3 \%$ étaient d'origine précambrienne et
Parc du Bic: location and place names.

$53,7 \%$, d'origine appalachienne. Le tableau I donne le pourcentage des différentes lithologies. Dans le groupe des précambriens prédominent les erratiques de la famille du granite $(41,6 \%)$ avec quelques blocs d'anorthosite $(2,7 \%)$. Les éléments appalachiens sont dominés par des lithologies locales, comme les conglomérats à clastes calcaires $(42,8 \%)$ et les grès-grauwacke $(36,3 \%)$ mais ils comprennent aussi des grès quartzitiques ou orthoquartzites $(8,1 \%)$ provenant d'affleurements sis en amont.

Il existe peu de différences entre les deux secteurs inventoriés, même si, sur le terrain, on observe certaines concentrations des plus gros blocs sur la batture argileuse à proximité du mont Chocolat et des deux îlots rocheux sis entre l'île Ronde et le mont Chocolat. En effet, dans la zone du marais à Spartine alterniflore, les précambriens totalisent $46,6 \%$ des méga-blocs, alors qu'ils comptent pour $45,8 \%$ dans le secteur de la batture dénudée. Le tableau II donne le pourcentage des diverses lithologies laurentidiennes et appalachiennes dans les deux secteurs.

Les pourcentages obtenus diffèrent sensiblement de ceux de Tremblay (1967, tabl. 1, p. 408), qui signale 70,5\% d'éléments laurentidiens pour les blocs de plus de $100 \mathrm{~cm}$ dans le secteur du marais, et $50,5 \%$ dans le secteur de la batture dénudée. Bien que ce secteur ne corresponde pas exactement à celui que nous avons étudié, le pourcentage de Tremblay est voisin de celui que nous avons obtenu $(54,2 \%)$; par contre, le pourcentage qu'il a calculé pour le marais est fort différent du nôtre.

\section{TAILLE ET POIDS DES MÉGA-BLOCS}

N'ont été retenus que les blocs dont le grand axe (Iongueur) excédait $100 \mathrm{~cm}$. Ces blocs situés à la surface de la batture peuvent être facilement mesurés et sont susceptibles d'être déplacés, à court terme, par des radeaux de glace. 


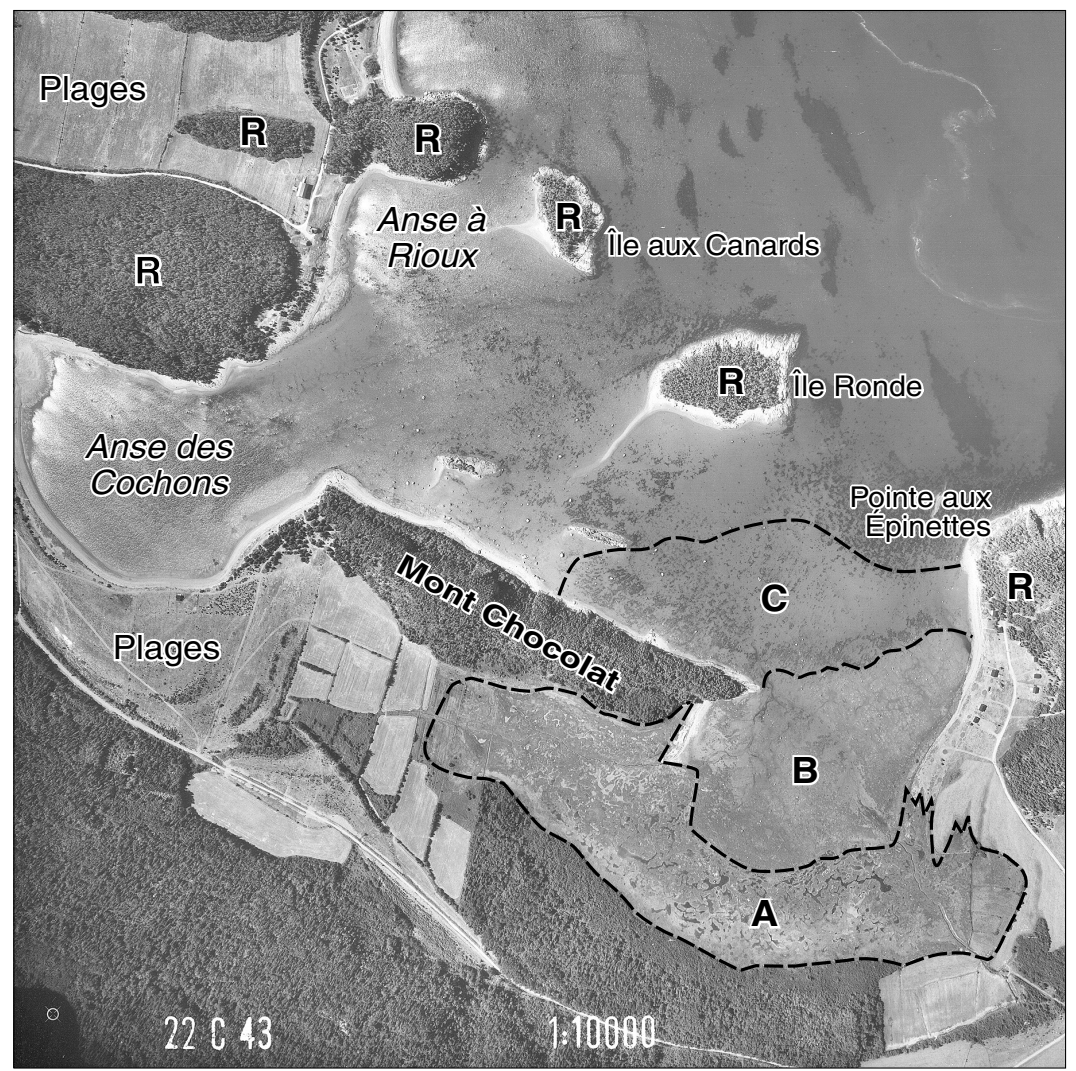

FIGURE 2. Photographie aérienne du secteur sud de la baie à l'Orignal, montrant le secteur étudié. Photo Q 76120-182, Photothèque québécoise. $A$, schorre supérieur; $\mathrm{B}$, schorre inférieur à méga-blocs épars; $C$, battures argileuse à méga-blocs; R, roc.

Airphotograph of the southern area of the Baie à l'Orignal showing the study area. Photo no 76120-182, Québec Photo-library. A, high marsh; $B$, low marsh with scattered mega-boulders; $C$, clayey tidal flat with mega-boulders; $R$, bedrock.

Sur les 88 méga-blocs mesurés dans le secteur du marais, il y avait 41 erratiques laurentidiens (précambriens); les deux plus gros mesurent respectivement $250 \times 185 \times 165 \mathrm{~cm}$ et $215 \times 160 \times 140 \mathrm{~cm}$ et les deux plus petits, $160 \times 115 \times 60 \mathrm{~cm}$ et $210 \times 95 \times 60 \mathrm{~cm}$.

Les 33 méga-blocs laurentidiens mesurés dans le secteur de la batture dénudée sont, dans l'ensemble, un peu plus petits. Les deux plus gros mesurent, en effet, $230 \times 120 \times$ $130 \mathrm{~cm}$ et $250 \times 190 \times 125 \mathrm{~cm}$ et les deux plus petits, $115 \times$ $70 \times 50 \mathrm{~cm}$ et $165 \times 85 \times 45 \mathrm{~cm}$.

Quant au poids (tabl. III), on constate que le plus petit bloc dans le marais a un poids estimé de 2,1 tonnes et le plus gros, de 14,3 , alors que la médiane est de 4,8 tonnes. Sur la batture dénudée, le poids du plus petit bloc est d'une tonne seulement et celui du plus gros, de 11,3 tonnes, alors que la médiane est de 3,5 tonnes.

En pourcentage par catégories, $51,2 \%$ des méga-blocs pèsent entre 2 et 5 tonnes dans le secteur du marais, alors que sur la batture dénudée, $75,6 \%$ des blocs pèsent entre 1 et 5 tonnes. Il y a donc une différence importante dans la taille des erratiques laurentidiens (précambriens) entre les deux secteurs. Qu'en est-il des appalachiens?

Dans le secteur du marais, le poids minimal des méga-blocs appalachiens mesurés est de 3,1 tonnes, le poids maximal, de 27,2 tonnes, et la médiane, de 6,6 tonnes, alors que sur la batture dénudée, le plus petit bloc pèse 1,6 tonnes, le plus gros, 76 tonnes, et la médiane est 8 tonnes.

En pourcentage par catégories, pour le secteur du marais, $83 \%$ de blocs appalachiens pèsent entre 1 et 10 tonnes, alors que sur la batture, le pourcentage tombe à $51,3 \%$; par contre, dans ce secteur, $35 \%$ des blocs pèsent entre 10 et 40 tonnes et $12,8 \%$ ont un poids supérieur à 40 tonnes. Le tableau IV donne la taille, le poids et la lithologie des dix plus gros blocs dans chaque secteur. La plus grande taille des méga-blocs appalachiens situés dans la zone de la batture dénudée est attribuable à la proximité des crêtes rocheuses, sources des blocs géants observés à faible distance des affleurements. En général, les blocs appalachiens sont anguleux et subanguleux, alors que les erratiques précambriens sont en majorité subarrondis.

\section{MOBILITÉ DES MÉGA-BLOCS}

La majorité des méga-blocs mesurés dans les deux secteurs reposaient directement sur la surface de la batture. Ils sont ainsi susceptibles d'être déplacés par les glaces lors du déglacement ou encore par des radeaux poussés par le vent et la marée lors de l'englacement. Bien qu'il existe des indices de déplacement de l'ordre du mètre dans l'un et l'autre secteur, 

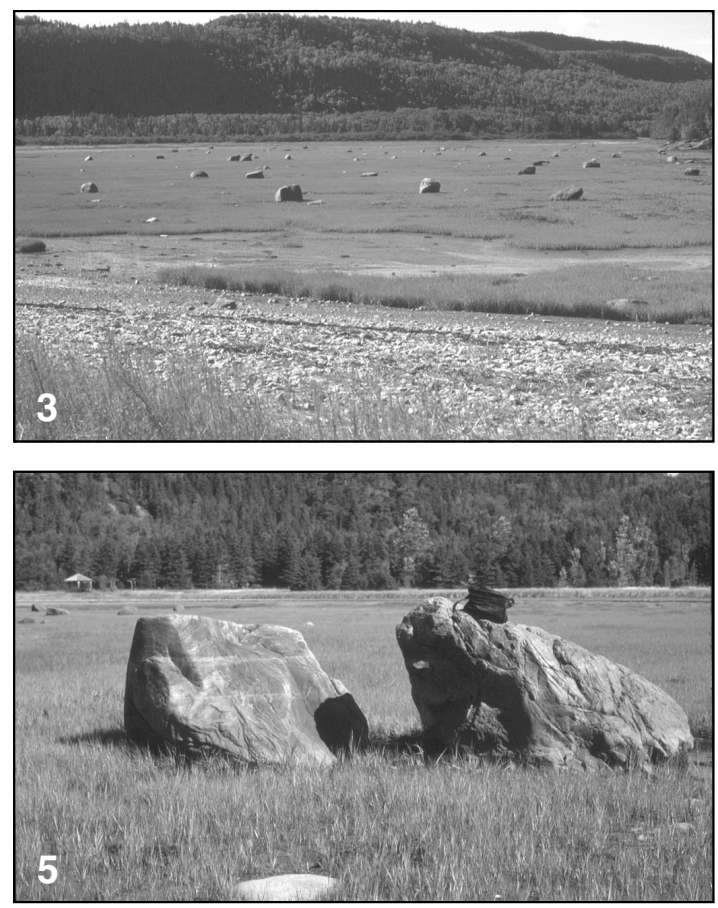

FIGURE 3. Vue générale du marais (schorre inférieur) à Spartine alterniflore avec des méga-blocs disséminés.

FIGURE 4. Méga-bloc de conglomérat calcaire à la surface du schorre inférieur à Spartine alterniflore. Le bloc mesure 240 × 230 × $160 \mathrm{~cm}$ et pèse environ 16,5 tonnes.

FIGURE 5. Deux méga-blocs à la surface du schorre inférieur à substrat argileux; celui de gauche est un grès quartzitique (appalachien) mesurant $240 \times 135 \times 130 \mathrm{~cm}$ et pesant environ 7,3 tonnes; celui de droite est un granite (laurentidien) mesurant $205 \times 115 \times 125 \mathrm{~cm}$ et pesant environ 5,5 tonnes.

FIGURE 6. Méga-bloc anguleux de grès (lithologie locale) sur la batture argileuse en face du mont Chocolat; le bloc mesure 450 x 320 x $230 \mathrm{~cm}$ et pèse environ 62 tonnes.

\section{TABLEAU I}

Lithologie des méga-blocs par catégorie

\begin{tabular}{lrlr}
\hline $\begin{array}{l}\text { Laurentidiens ou } \\
\text { précambriens }\end{array}$ & $\%$ & Appalachiens & $\%$ \\
\hline Granite & 41,6 & Grès quartzitique & 8,1 \\
Gneiss & 30,5 & Grès-grauwacke & 36,3 \\
Granito-gneiss & 4,2 & Grès et schiste & 1,1 \\
Arnorthosite & 2,7 & Grès et conglomérat à petits quartz & 3,2 \\
Autres & 21 & Conglomérat calcaire (local) & 42,8 \\
& & Conglomérat et grès calcaire (local) & 7,4 \\
& & Conglomérat à petits quartz & 1,1 \\
\hline
\end{tabular}

Nombre total de blocs : 160 , dont 74 laurentidiens $(46,3 \%)$ et 86 appalachiens $(53,7 \%)$
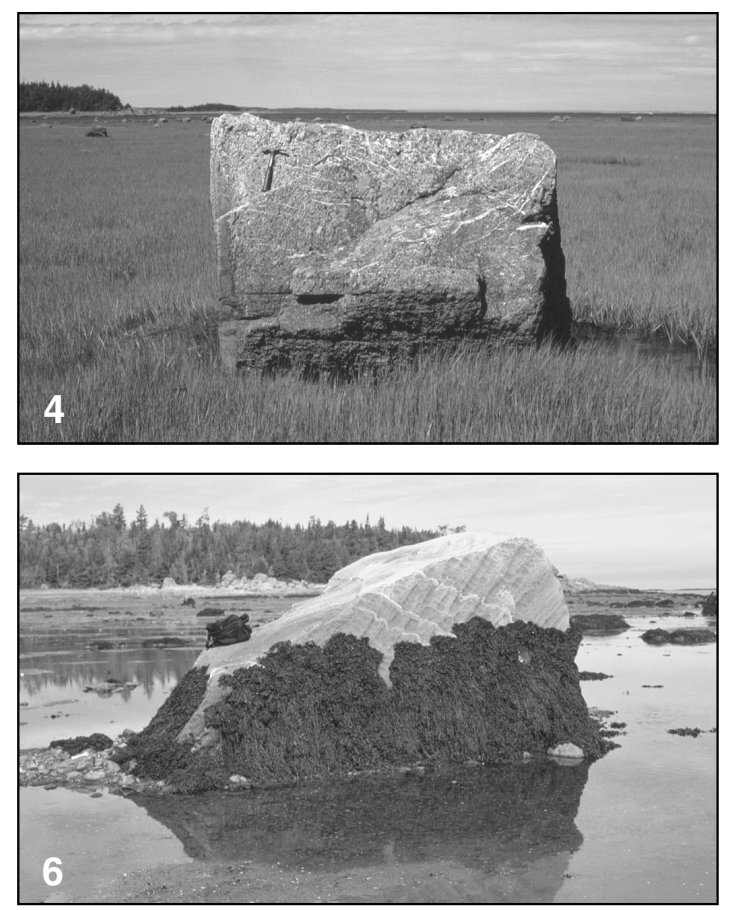

A general view of the salt-water cordgrass (Spartina alterniflora) with scattered mega-boulders.

A polymictic calcareous conglomerate mega-boulder $240 \times 230 \times$ $160 \mathrm{~cm}$ weighing approximately 16.5 tons on the Spartina low marsh.

Two mega-boulders on the low marsh surface; the boulder to the left is an Appalachian quartzitic sandstone $(240 \times 135 \times 130 \mathrm{~cm})$ weighing approximately 7.3 tons; the other boulder is a Precambrian granite $(205 \times 115 \times 125 \mathrm{~cm})$ weighing 5.5 tons.

An angular sandstone mega-boulder $(450 \times 320 \times 230 \mathrm{~cm})$ weighing approximately 62 tons on the surface of the clayey tidal flat.

nous avons observé très peu de déplacements récents importants. Le secteur du marais étant mieux abrité, les glaces, sous l'influence des courants de marée et du vent, devraient y exercer une action plus modérée que sur la batture argileuse dénudée. Là, les plus petits méga-blocs, davantage exposés à la pression des radeaux de glace, devraient être plus facilement déplacés. Quant aux très gros blocs appalachiens, ils sont à toutes fins pratiques immobiles.

Compte tenu de la taille des blocs, les déplacements sont attribuables à la pression exercée par les radeaux de glace entraînés principalement par le jusant. Les blocs ont donc tendance à migrer du haut vers le bas de la batture. Les déplacements en sens contraire et latéraux sont occasionnels, ne comptant que pour environ $5 \%$. Pour cette raison, on n'observe pas de concentrations de méga-blocs sur le haut du rivage et leur répartition est plutôt éparse sur la batture. 
TABLEAU II

Lithologie des méga-blocs pour chaque secteur

\begin{tabular}{lrc}
\hline & $\begin{array}{c}\text { Marais } \\
\text { intertidal } \\
(\%)\end{array}$ & $\begin{array}{c}\text { Batture } \\
\text { dénudée } \\
(\%)\end{array}$ \\
\hline Laurentidiens ou précambriens & & \\
Granite & 31,7 & 51,5 \\
Gneiss & 36,6 & 24,3 \\
Granito-gneiss & 2,4 & 6,0 \\
Arnothosite & 2,4 & 3,0 \\
Autres & 26,8 & 15,2 \\
Total de la catégorie dans ce secteur & $(n-41) 46,6$ & $(n-33) 45,8$ \\
Appalachiens & & \\
Grès quartzitique & 8,5 & 7,7 \\
Grès-grauwacke & 21,3 & 51,3 \\
Grès et schiste & 2,1 & - \\
Grès et conglomérat à petits quartz & 6,4 & - \\
Conglomérat calcaire (local) & 57,4 & 28,2 \\
Conglomérat et grès calcaire (local) & 2,1 & 12,8 \\
Conglomérat à petits quartz & 2,1 & - \\
Total de la catégorie dans ce secteur & $(n-47) 53,4$ & $(n-39) 54,2$ \\
Nombre total de blocs du secteur & $(n-88)$ & $(n-72)$ \\
\hline
\end{tabular}

\section{TABLEAU III}

Poids des méga-blocs

\begin{tabular}{|c|c|c|}
\hline & Marais intertidal & Batture dénudée \\
\hline & \multicolumn{2}{|c|}{ Poids (tonnes) } \\
\hline \multicolumn{3}{|l|}{ Laurentidiens } \\
\hline Minimum & 2,1 & 1 \\
\hline Maximum & 14,3 & 11,3 \\
\hline Médiane & 4,8 & 3,5 \\
\hline \multicolumn{3}{|l|}{ Appalachiens } \\
\hline Minimum & 3,1 & 1,6 \\
\hline Maximum & 27,2 & 95 \\
\hline \multirow[t]{2}{*}{ Médiane } & 6,6 & 8 \\
\hline & \multicolumn{2}{|c|}{ Pourcentage } \\
\hline \multicolumn{3}{|l|}{ Laurentidiens } \\
\hline 1 à 3 tonnes & 14,6 & 45,5 \\
\hline 3 à 5 tonnes & 36,6 & 30,1 \\
\hline 5 à 10 tonnes & 46,3 & 18,2 \\
\hline 10 à 15 tonnes & 2,4 & 6,1 \\
\hline \multicolumn{3}{|l|}{ Appalachiens } \\
\hline 1 à 5 tonnes & 31,9 & 28,2 \\
\hline 5 à 10 tonnes & 51,1 & 23,1 \\
\hline 10 à 20 tonnes & 12,7 & 25,6 \\
\hline 20 à 30 tonnes & 4,3 & 2,6 \\
\hline$>30$ tonnes & - & 25,6 \\
\hline
\end{tabular}

\section{TABLEAU IV}

Les dix plus gros méga-blocs dans les deux secteurs inventoriés

\begin{tabular}{|c|c|c|}
\hline Taille (cm) & $\begin{array}{c}\text { Poids } \\
\text { (tonnes) }{ }^{*}\end{array}$ & Lithologie \\
\hline \multicolumn{3}{|l|}{ Marais intertidal } \\
\hline $345 \times 205 \times 205$ & 27,2 & Conglomérat calcaire \\
\hline $250 \times 240 \times 160$ & 18,0 & Conglomérat calcaire \\
\hline $245 \times 245 \times 150$ & 17,0 & Grès quartzitique \\
\hline $240 \times 230 \times 160$ & 16,5 & Conglomérat calcaire \\
\hline $250 \times 185 \times 165$ & 14,3 & Gneiss \\
\hline $230 \times 200 \times 160$ & 13,8 & Grès-grauwacke \\
\hline $300 \times 200 \times 100$ & 11,3 & Conglomérat calcaire \\
\hline $225 \times 190 \times 135$ & 10,8 & Conglomérat calcaire \\
\hline $275 \times 145 \times 130$ & 9,7 & Conglomérat calcaire \\
\hline $215 \times 160 \times 140$ & 9,1 & Gneiss \\
\hline \multicolumn{3}{|l|}{ Batture dénudée } \\
\hline $565 \times 350 \times 205$ & 76 & Conglomérat et grès \\
\hline $430 \times 260 \times 300$ & 63 & Conglomérat calcaire \\
\hline $450 \times 320 \times 230$ & 62 & Grès-grauwacke \\
\hline $525 \times 340 \times 160$ & 54 & Conglomérat calcaire \\
\hline $400 \times 300 \times 180$ & 41 & Grès \\
\hline $420 \times 200 \times 225$ & 36 & Conglomérat calcaire \\
\hline $500 \times 220 \times 155$ & 32 & Conglomérat calcaire \\
\hline $320 \times 265 \times 195$ & 31 & Grès \\
\hline $390 \times 300 \times 95$ & 21 & Conglomérat calcaire \\
\hline $330 \times 255 \times 120$ & 19 & Conglomérat calcaire \\
\hline
\end{tabular}

* Le poids théorique des blocs a été réduit de $25 \%$ pour tenir compte de la morphométrie.

\section{INDICES DE DÉPLACEMENT}

Des indices de déplacement plus ou moins récents (rainure arrière, cuvette, bourrelet du côté mer) ont été observés sur 28 blocs, soit $31,8 \%$ des 88 méga-blocs mesurés dans le secteur du marais. Dans ce domaine, les deux groupes sont à peu près égaux, soit $29,3 \%$ pour les blocs laurentidiens et $34 \%$ pour les appalachiens.

La valeur des déplacements (tabl. V) est plutôt faible en comparaison de celle observée dans d'autres sites de la rive sud (Dionne, 1988, 2001a). Le plus grand déplacement s'est fait sur $6 \mathrm{~m}$ et a touché un bloc laurentidien de 6,3 tonnes. Deux autres précambriens, de 14,3 et 5,8 tonnes respectivement, ont été déplacés sur $4 \mathrm{~m}$ et trois blocs (1 précambrien et 2 appalachiens), sur $3 \mathrm{~m}$.

Rappelons que le secteur du marais, sis à la partie supérieure de la batture, est une zone relativement protégée où l'action des glaces est de faible à modérée.

Curieusement, nous n'avons pas observé de traces de déplacement récent autour des méga-blocs de la batture dénudée, bien que plusieurs blocs reposant directement sur la 
TABLEAU V

Méga-blocs déplacés

\begin{tabular}{lcc}
\hline Lithologie & $\begin{array}{c}\text { Poids } \\
\text { (tonnes) }\end{array}$ & $\begin{array}{c}\text { Déplacement } \\
(\mathrm{m})\end{array}$ \\
\hline Gneiss & 6,3 & 6 \\
Igné de couleur foncée & 5,8 & 4 \\
Gneiss & 14,3 & 4 \\
Granite & 5,7 & 3 \\
Conglomérat calcaire & 5,6 & 3 \\
Grès-grauwacke & 7,6 & 3 \\
Conglomérat calcaire & 6 & 2,5 \\
Métamorphique & 8,9 & 1,5 \\
Métamorphique & 7,7 & 1,5 \\
Grès & 4 & 1,5 \\
Grès & 6,8 & 1,5 \\
Conglomérat calcaire & 5,5 & 1 \\
Granite & 6,6 & 1 \\
Métamorphique & 4 & 1 \\
\hline
\end{tabular}

surface aient pu être déplacés récemment et que quelquesuns, inclinés vers la rive, portent des signes de pressions exercées par des radeaux de glace entraînés vers le large par le jusant. Il est possible, toutefois, que les traces superficielles de déplacement aient été effacées par l'action des vagues et des courants au cours de l'interglaciel, les observations ayant été faites en septembre. Ceci semble confirmé par un nouveau relevé fait en mai 2003, au cours duquel nous avons constaté que plusieurs méga-blocs du schorre et de la batture avaient été déplacés.

Dans le secteur du marais, Tremblay (1967, tabl. 1, p. 408) signale avoir observé 200 blocs d'un mètre et plus dont 141 erratiques laurentidiens et 59 appalachiens. Pour notre part, dans l'ensemble du marais, mais en particulier dans la partie inférieure où sont dispersés la très grande majorité des méga-blocs, nous n'avons mesuré que 41 précambriens et 47 appalachiens de cette taille pour un total de 88. II en manquerait donc 112 par rapport aux chiffres fournis par Tremblay. Où sont-ils passés?

Nous proposons deux explications : a) ou bien nous avons négligé de mesurer de nombreux blocs dont le grand axe était légèrement supérieur à $100 \mathrm{~cm}$, estimant visuellement que leur taille était trop petite pour les ranger dans la catégorie des méga-blocs, ce qui nous paraît peu vraisemblable; b) ou bien, plus de la moitié (56\%) des blocs d'une longueur supérieure à $100 \mathrm{~cm}$ identifiés par Tremblay en 1965 ont été déplacés depuis. Cette explication implique une très grande mobilité des méga-blocs du marais, mobilité peu plausible compte tenu des nombreuses observations faites depuis 40 ans sur l'ensemble de la rive sud (Dionne, 1988) et du fait que les quelques méga-blocs localisés dans le schorre supérieur peuvent difficilement, eux, être déplacés par les glaces.

\section{CONCLUSION}

Les méga-blocs situés à la surface de la batture argileuse de la baie à l'Orignal, dans le parc du Bic, ne manquent pas d'attirer l'attention des visiteurs. Dans le secteur du marais à Spartine alterniflore, les blocs sont d'accès facile à marée basse. Leur examen permet de distinguer l'erratique laurentidien du bloc appalachien provenant des crêtes rocheuses dans le parc du Bic et de constater la présence ou l'absence d'indices de déplacement. On sait maintenant que plus de $46 \%$ des méga-blocs de la batture aux environs de la pointe du Glaciel sont des erratiques provenant du Bouclier laurentidien, situé à plus de $30 \mathrm{~km}$ sur la rive nord du Saint-Laurent. On sait aussi que ces cailloux ont été transportés par des icebergs plutôt que par les glaciers, d'autant plus que vers la fin de la dernière période glaciaire, dite du Wisconsinien, l'écoulement des glaces appalachiennes se faisait vers le NO, puis vers le $\mathrm{N}$, et finalement vers le NE. À plusieurs endroits dans le parc du Bic, on peut observer sur le rivage rocheux des stries et autres marques d'érosion montrant que le dernier écoulement se faisait vers le NE.

À l'exception des plus gros, les blocs disséminés à la surface de la batture sont très mobiles et sont facilement déplacés par les radeaux de glaces qui exercent de grandes pressions lorsqu'ils sont entraînés par le jusant ou poussés par le vent. De façon générale, les blocs libres migrent vers le large. L'activité glacielle peut être observée principalement lors du déglacement.

\section{REMERCIEMENTS}

Nous remercions M. Pierre J.H. Richard pour sa lecture critique du manuscrit.

\section{RÉFÉRENCES}

Brochu, M. et Michel, J.-P., 1994. Dictionnaire de géomorphologie à caractère dimensionnel. Guérin Éditeur, Montréal, 298 p.

Dionne, J.-C., 1963. Pour l'emploi rationnel des termes géographiques appliqués aux littoraux de l'Arctique canadien. Le Géographe canadien, 7 : 116-130. 1966. Carte morpho-sédimentologique de la région de Rimouski (22 C/7). Bureau d'Aménagement de l'Est du Québec, Mont-Joli, et Office de planification et de développement du Québec, Québec, échelle du 1/50 000.

1972. Caractéristiques des blocs erratiques des rives de l'estuaire du Saint-Laurent. Revue de Géographie de Montréal, 26 : 125-152.

1979. Les blocs d'estran à Saint-Fabien-sur-Mer, estuaire maritime du Saint-Laurent, Québec. Maritime Sediments, $15: 5-13$.

1988. Ploughing boulders along shorelines with particular reference to the St. Lawrence estuary. Geomorphology, 1 :297-308.

2001a. Observations géomorphologiques sur les méga-blocs d'un schorre à Spartina alterniflora du Saint-Laurent, Québec. Géomorphologie, 7 : 243-255. 2001b. Le tombolo du cap Enragé, parc du Bic, Bas-Saint-Laurent. Géographie physique et Quaternaire, 55 : 181-191.

2002a. Les erratiques de dolomie à Rivière-Blanche, côte sud de l'estuaire maritime du Saint-Laurent : un indicateur de transport glaciaire et glaciel. Canadian Journal of Earth Sciences, 39 : 1239-1255.

2002b. The boulder barricade at Cap à la Baleine, north shore of Gaspé Peninsula (Québec): Nature of boulders, origin and significance. Journal of Coastal Research, $18: 652-661$.

Dionne, J.-C. et Poitras, S., 1998a. Lithologie des cailloux de la baie de Mitis, rive sud de l'estuaire maritime du Saint-Laurent : un exemple de transport glaciaire et glaciel complexe. Géographie physique et Quaternaire, 52 : 107-122. 
1998b. Geomorphic aspects of mega-boulders at Mitis Bay, Lower St. Lawrence estuary, Québec. Journal of Coastal Research, 14 : 1054-1064.

Favereau, R., 1988. Synthèse de la géologie et de la géomorphologie du parc du Bic. Ministère du Loisir, de la Chasse et de la Pêche, Service du plein air et des parcs, Québec, 118 p.

Fortin, D. et Belzile, L., 1996. Le parc du Bic. Édition du Trécarré, Saint-Laurent, $89 \mathrm{p}$.

Genest, C.G., 2000. Dictionnaire de géomorphologie. Société de géographie de la Mauricie, Trois-Rivières, $411 \mathrm{p}$.

Lajoie, J., 1971. Région de Rimouski et de Lac-des-Baies (moitié ouest), comtés de Rimouski et de Rivière-du-Loup. Ministère des Richesses naturelles, Québec, Rapport manuscrit DP-0064, 41 p. + cartes hors texte.
Québec, 1987. Répertoire toponymique du Québec. Les Publications du Québec, Québec, 1900 p.

1994. Noms et lieux du Québec : dictionnaire illustré. Commission de toponymie du Québec, Les Publications du Québec, Québec, 925 p.

Rappol, M., 1993. Ice flow and glacial transport in Lower St. Lawrence, Québec. Geological Survey of Canada, Ottawa, Paper 90-19, 28 p.

Tremblay, G., 1967. Observations et mesures sur les blocs glaciels du cap à l'Orignal. Cahiers de Géographie de Québec, 11 : 402-411.

1986. Parc du Bic : étude géomorphologique. Département de Géographie, Université Laval. Rapport présenté au Ministère du Loisir, de la Chasse et de la Pêche, Québec, 100 p. + annexes.

Tremblay, V., 1947. La question de «Baie des Hahas». Société historique du Saguenay, Bulletin, $5: 2-14$

\section{APPENDICE}

\section{REMARQUES SUR LA TOPONYMIE}

Des efforts louables ont été faits ces dernières années pour améliorer la toponymie du parc du Bic. II reste toutefois encore plusieurs entités d'intérêt géographique à nommer. En tant que géographe et géomorphologue professionnel ayant une connaissance approfondie des divers aspects morpho-sédimentologiques du rivage du parc et en ma qualité d'auteur ayant déjà soulevé la question des génériques dans l'Arctique canadien (Dionne, 1963), on me permettra deux observations : l'une concerne les génériques et l'autre, quelques toponymes.

En ce qui a trait aux génériques, dans la dernière version de la carte du parc du Bic publiée par Parcs Québec, le plus grand des trois principaux rentrants s'appelle « anse à l'Orignal » et son littoral comprend lui-même plusieurs petits rentrants, appelés eux aussi «anses». Dans le coin SO, cependant, le rentrant porte le nom de «baie des Cochons». Il est difficile de comprendre la logique de ces deux désignations, d'autant que sur la version antérieure de la carte, on peut lire «baie à l'Orignal» et «anse des Cochons». Pourquoi avoir modifié les anciens génériques qui correspondaient à la réalité ? Au besoin, on se reportera à l'ouvrage de Brochu et Michel (1994) et à celui de Genest (2000) pour comprendre la distinction entre une baie et une anse. Mais en général, la plupart des gens savent qu'une anse est un rentrant plus modeste et moins accentué qu'une baie. On ne saurait donc trouver une baie dans une anse, ce qui est pourtant le cas sur la dernière carte du parc du Bic.

En ce qui a trait à la toponymie, la première remarque concerne l'anse des Pilotes. Sur l'ancienne carte et dans le livre de Fortin et Belzile (1996), les deux anses sises de part d'autre du tombolo du cap Enragé portaient des noms différents : «anse des Pilotes » du côté ouest, et «anse aux Bouleaux » du côté est. Sur la nouvelle version, on peut lire «anse aux Bouleaux ouest et est ». Le remplacement du toponyme «anse des Pilotes » aurait apparemment comme fondement l'existence à l'île du Bic d'une seconde anse des Pilotes. Nous doutons de la valeur de cet argument quand on sait qu'il existe au Québec 158 lacs Vert, 133 lacs Noir, 186 lacs Rond, 138 lacs des îles, etc. Par ailleurs, les deux «anses des Pilotes » sont signalées dans Le répertoire toponymique du Québec (Québec, 1987, p. 1388). L'anse des Pilotes étant depuis plusieurs années un important site d'observation des phoques, on aurait pu l'appeler «anse aux Phoques ", s'il était vraiment nécessaire de modifier la toponymie du lieu.

La deuxième remarque se rapporte à l'anse à Mercier. À Saint-Fabien-sur-Mer, la petite anse du secteur NE ou du côté sud de l'îlet au Flacon porte le nom d'«anse à Mercier », soit le même que l'ensemble du rentrant. Pour distinguer ce petit rentrant du grand, nous l'avons depuis longtemps appelé «anse au Flacon », ce qui permet de le situer plus rapidement.

La troisième remarque concerne la graphie de la baie du $\mathrm{Ha}$ !Ha!. Plusieurs se sont interrogés sur l'origine du nom «Ha!Ha! » que l'on retrouve ailleurs au Québec (lac, rivière et baie des Ha! Ha!, au Saguenay et dans les Laurentides, Saint-Louis du Ha!Ha!, au Témiscouata, et baie du Ha!Ha!, sur la basse Côte-Nord, près de la Tabatière). Diverses explications ont été proposées, mais peu sont convaincantes. À notre connaissance, seul monseigneur Tremblay (1947), historien saguenéen, a abordé la question sérieusement et a proposé la graphie moins exclamatoire de «baie des Hahas», au Saguenay, et «baie du Haha», dans le parc du Bic. Dans le bel ouvrage abondamment illustré Noms et Lieux du Québec (Québec, 1994, p. 265-266), on mentionne pourtant l'existence de «l'Isle du Haha» sur la carte de la NouvelleFrance (Acadie) de Franquelin (1686) et celle de la «baie du Haha » sur la côte nord, sur la carte de Boishébert (1715). Apparue beaucoup plus tard, la graphie Ha!Ha! ne devraitelle pas être remplacée par l'originale? Nous le souhaitons.

Notre quatrième remarque porte sur l'île à d'Amours. Sur la version récente de la carte du parc du Bic, on a retenu le nom de «l'île aux Amours" en remplacement de «l'île à d'Amours », ce qui est plus romantique, colle davantage à la réalité et correspond au toponyme de la carte topographique (feuillet 22C/7 est) datant du début des années 1960. Enfin, soulignons en passant que sur la nouvelle carte du parc du Bic, la pointe NE du mont Chocolat porte le joli nom de «pointe du Glaciel ». 